In one instance of an inflammatory condition of an eye the diagnosis was made on the strength of a weakly positive complement fixation test for gonorrhoea in the blood serum. As evidence of infection this result was negligible, but it led to dire consequences for the patient, including the, fortunately temporary, break-up of his marriage. Another man accused his wife of unfaithfulness because she was found to have trichomonal vaginitis and this was stigmatized as "V.D." In yet another instance a woman with " a lesion on the right upper lid resembling a rodent ulcer" was informed that she had syphilis. Serological tests for syphilis were in fact negative, but the doctor jumped to a hasty conclusion because the results were entered in her notes in red ink.

Seale $^{2}$ has reported the case of a young woman who committed suicide because her boy friend had non-gonococcal urethritis and she understood it to be a venereal disease. These are extreme instances, but others of the same nature are not uncommon in venereological practice. They cause untold harm to individuals and their families, and there is no possible justification for them. It should be a golden rule that suspicions of this kind should never be communicated to patients unless and until they become reasonable certainties; and it is equally important that treatment should not be undertaken until the diagnosis has been confirmed. Otherwise the true diagnosis may never be known and suspicions and anxieties once implanted may be impossible to eliminate. Even in these days of scientific progress the fundamental rules are valid. One is usually rendered in Latin as "Primum non nocere," and the second equally briefly in English, "Diagnosis before treatment."

\section{Treatment of Thyrotoxicosis}

There can be few disorders in which the choice of treatment can be so delicately balanced as in thyrotoxicosis. The balance lies between subtotal thyroidectomy, prolonged administration of antithyroid drugs, and radioiodine therapy. Although the absolute indications for any one of these methods are comparatively few, the relative pros and cons are numerous and tend to vary with time, prejudice, and the local climate of opinion. In practice the choice is more often determined by available facilities, the experience of the clinician in charge, and the wishes of the patient.

Subtotal thyroidectomy, first advocated by Kocher in 1895, was the only effective treatment until the advent of radioiodine in 1942 . The hazards of postoperative thyroid crisis became less after 1923 with the preoperative use of Lugol's iodine and were virtually abolished when routine preparation with antithyroid drugs was adopted. The risks of damage to the recurrent laryngeal nerves and parathyroid glands, though still important, have lessened with improved surgical techniques in good hands.

The advantages of subtotal thyroidectomy are a relatively low incidence of subsequent hypothyroidism or of a recurrence of thyrotoxicosis. Indeed, it is remarkable how so crude and empirical a procedure as resection of about seveneighths of the thyroid gland leads to a euthyroid state in the majority of thyrotoxic patients. Surgery is specially indicated in the case of large or nodular goitres, in single toxic adenomata as defined by a radioiodine scan, in rare cases of intrathoracic goitre, and when antithyroid drugs have failed or cannot be tolerated. In children subtotal thyroidectomy leads to a higher incidence of hypothyroidism than in adults. Children, therefore, are best treated with antithyroid drugs until well after puberty. Other contraindications are pregnancy, severely ill patients, and the recurrence of thyrotoxicosis after previous subtotal thyroidectomy. In this last situation the risks of causing damage to recurrent laryngeal nerves and parathyroid glands are much higher. If malignant exophthalmos is threatened or present it is often aggravated by thyroidectomy and is best treated with antithyroid drugs. Lastly, the risks of a disfiguring keloid scar forming at the site of operation are much higher in young adults than in older patients; so this factor may influence the choice of treatment.

At page 643 of this week's issue of the B.M.F. Mr. A. D. McNeill and Dr. J. A. Thomson review the long-term results of subtotal thyroidectomy in 123 patients. As is usual there were no operative deaths, though immediate complications of a benign type occurred in about $10 \%$ of patients. Eventually hypothyroidism developed in about $7 \%$ of patients, which accords with average experience; thyrotoxicosis recurred in about $11 \%$, though in one-third of these it was mild enough to need no treatment. These authors draw attention to an inverse relation between these two complications in different reported series: probably this depends on the amount of thyroid tissue removed by different surgeons.

By far the most serious complications of subtotal thyroidectomy are permanent damage to recurrent laryngeal nerves, or the parathyroid glands, or both. In this series almost $6 \%$ of patients were found on laryngoscopy to have suffered damage to the vocal cords, and about $10 \%$ admitted to a change of voice. This suggests that this operation is probably best avoided in those whose livelihood depends on their voice. Permanent hypoparathyroidism occurred in only one patient, though two others showed symptoms and biochemical signs of parathyroid deficiency, which recovered within two years. If it passes unrecognized and untreated, hypoparathyroidism gives rise to much general ill-health, and it predisposes to the development of cataracts. Its treatment demands careful and lifelong supervision.

Radioiodine, though much slower in correcting thyrotoxicosis than subtotal thyroidectomy or antithyroid drugs, seemed at first to offer the ideal method of treatment in patients over 40 years in age. In younger age groups its use has been precluded, at least in Britain, for fear of possible late carcinogenic effects, though these fears so far have proved unfounded. There is still no certain way of estimating the correct dose of radioiodine in relation to size, nodularity, and activity of the thyrotoxic gland. Too small a dose increases the risks of recurrence and the need for further treatment, whereas too large a dose predisposes to early hypothyroidism. Nodular toxic goitres tend to need larger doses than diffuse toxic goitres, and are often best treated surgically, especially if giving rise to pressure symptoms. The most serious objection to radioiodine therapy has come to be appreciated only with the passage of time. This is a progressive and relentless rise in the incidence of hypothyroidism with each year after treatment. This shows no signs of levelling off and has already reached an incidence of

- Green, M., and Wilsun, G. M., Brit. med. f., 1964, 1, 1005.

2 Greene, R and Morgan, D. C F clin. Endocrin, 1956, 16, 391.

s Hershman, J. M., Givens, J. R., Cassidy, C. E., and Astwood, E. B., f. clin. Endocrin, 1966, 26, 803 . 
$35 \%$ or more after ten years. ${ }^{1}$ Many patients will have become hypothyroid so insidiously that this has been overlooked. Thus it is necessary to follow up patients treated with radioiodine indefinitely, for sooner or later a large proportion of them will need permanent treatment with thyroxine. Awareness of this late effect has undoubtedly tipped the balance in favour of treatment by surgery or by a prolonged course of antithyroid drugs.

Antithyroid drugs came into use about 20 years ago, first as thiouracil derivatives. Propylthiouracil, which is still the most popular of these drugs in the United States, proved much less toxic than methylthiouracil. In Britain carbimazole has long been the drug of choice and is thought to be even less toxic. With its use in proper dosage the incidence of blood dyscrasias such as agranulocytosis and thrombocytopenic purpura is extremely small. Skin reactions-which occur in about $4 \%$ of treated patients ${ }^{2}-$ are often mild enough to be controllable with antihistamines, or are only transient. As with the thiouracil derivatives, toxic effects occur mainly during the first month of treatment and seldom thereafter, a feature which favours the long-term use of these drugs.

There are few absolute contraindications to this form of treatment, when minimal effective doses are used under careful supervision. These are, firstly, the presence of large or intrathoracic goitres which are causing or are apt to cause pressure symptoms, and, secondly, intolerance of the drug. The former is more likely to occur with overdosage, and the latter can often be overcome by switching from carbimazole to propylthiouracil or vice versa. Nevertheless, even with carefully controlled dosage and concurrent administration of thyroxine, some goitres in severely thyrotoxic young patients may continue to enlarge, in which case surgery is indicated. Surgery is also preferable in patients of low intelligence or disturbed mind who cannot be relied upon to take the drugs at regular intervals.

Antithyroid drugs are specially indicated in cases of mild to moderate severity, in children, during pregnancy (provided the dose is kept to a minimum), in relapse after previous subtotal thyroidectomy, and when there is a threat of malignant exophthalmos. A recent survey of 176 patients $^{3}$ showed prolonged remission after one course of treatment in half of the cases. Relapse occurred mainly during the first year after treatment, and in only $3 \%$ after six years. With repeated courses the ultimate rate of remission was about $75 \%$ over 10 to 20 years. The shorter the course of treatment and the more severe the illness the more likely is relapse to occur. It is usual to give the drugs for a period of one to two years at a minimal effective dose before gradually withdrawing treatment.

\section{Tropical Ataxic Neuropathy}

Our study of diseases of the nervous system in the tropics is now in the stage which the neurology of temperate climates reached about 50 years ago-namely, recognition, description, and analysis of disorders and their correlation with the underlying pathological lesions. ${ }^{1}$ Though recent work has suggested that certain obscure tropical neurological syndromes such as kuru, ${ }^{2}$ which has been observed in New Guinea, and possibly the Parkinsonism-dementia complex occurring in the
Chamorro population in the Mariana Islands, may possibly be the result of slow virus infections, certain other neurological syndromes commonly observed in tropical countries have been widely assumed to be of nutritional origin.

The symptoms of these so-called nutritional neuropathies have included amblyopia (attributed to bilateral retrobulbar neuritis leading to optic atrophy), bilateral nerve deafness, myelopathy involving the pyramidal tracts of the spinal cord but even more severely the posterior columns, and polyneuropathy, occurring either singly or in combination in the same individual. H. Strachan ${ }^{3}$ and H. H. Scott ${ }^{4}$ were among the first to describe such a syndrome in the inhabitants of Jamaica, and more recently the condition has been extensively studied in Jamaica, ${ }^{5}$ Sierra Leone, ${ }^{6}$ Liberia, $^{7}$ Nigeria, ${ }^{89}$ Tanganyika, ${ }^{10}$ and Senegal. ${ }^{11}$

In a recent thorough study of 84 cases of this syndrome, for which the title "tropical ataxic neuropathy" has been generally accepted, B. O. Osuntokun ${ }^{1}$ showed that the sex incidence is equal and that the highest incidence is in patients aged 30 to 50 of poor social and nutritional status. The commonest symptoms are sensory, initially confined to the legs, with an ataxic gait and less constant visual impairment, weakness of the lower limbs, tinnitus, deafness, and recurrent mucocutaneous lesions usually associated with ariboflavinosis. In his series the commonest findings on physical examination were sensory loss of "posterior column" type, sensory ataxia, a positive Romberg's sign, bilateral optic atrophy, perceptive deafness, and wasting and weakness in the legs in that descending order. While similar symptoms have been described in West Indian patients, some have been reported to be suffering from amblyopia only, ${ }^{12}$ while in another series $^{13}$ there was a high incidence of spasticity of the lower extremities due to lesions of the pyramidal tract as well as evidence of sensory loss of posterior-column type. In the Nigerian cases extensor plantar responses and superficial sensory loss were less common. In general, the cerebrospinal fluid is normal; a high incidence of positive Wassermann reactions in the fluid in the West Indian cases was probably coincidental. Nutritional studies in these families have usually shown normal protein nutrition but a subnormal calorie intake. Intake of riboflavine and to a less extent of thiamine has been low as measured by urinary excretion, but the serum levels of folate and vitamin $B_{12}$ are generally normal or high. Electrodiagnostic tests have generally given evidence of polyneuropathy even in patients in whom the spinal cord was chiefly affected.

This week in the B.M.f. Dr. Osuntokun and his colleagues describe at page 647 a study of nine cases, and confirm that the patients had all had an inadequate diet made up very largely of cassava derivatives. Cassava, the tuber of manioc, contains a cyanogenetic glycoside (linamarin), from which cyanide is released on hydrolysis. Dr. Osuntokun and his colleagues found much-reduced levels in the plasma of

Osuntokun, B. O., Brain, 1968, 91, 215.

2 Mathews, J. D., Glasse, R., and Lindenbaum, S., Lancet, 1968, 2, 449. - Strachan, H., Practitioner, 1897, 59, 477.

- Scot, H. H., Ann. trop. Med. Parasit., 1918, 12, 109.

- Cruickshank, E. K., Montgomery, R. D., and Spillane, J. D., Wld Neurol., 1961, 2, 199.

- Rowland, H. A. K., F. trop. Med. Hyg., 1963, 66, 181

' Knüttgen, H., Z. Tropenmed. Parasit., 1955, 6, 472.

- Money, G. L., W. Afr. med. $\} ., 1959,8,3$.

- Monekosso, G. L., W. Indian med. F., 1962, 11, 240.

10 Latham, M. C., Brit. F. Nutr., 1964, 18, 129.

Collomb, H., Quere, M. A., Cros, J., and Giordano, C., f. neurol. Sci., $1967,5,159$.

12 MacKenzie, A. D., and Phillips, C. I., Brain, 1968, 91, 249.
13 Montgomery, R. D., Cruickshank, E. K., Robertson, W. B., and McMenemey, W. H., Brain, 1964, 87, 425. 